\title{
Inhibition of murine K-BALB and CT26 tumour growth using a Semliki Forest virus vector with enhanced expression of IL-18
}

\author{
C.P. CHIKKANNA-GOWDA ${ }^{1}$, S. McNALLY ${ }^{1}$, B.J. SHEAHAN ${ }^{2}$, M.N. FLEETON $^{1}$ and G.J. ATKINS ${ }^{1}$ \\ ${ }^{1}$ Department of Microbiology, Moyne Institute, Trinity College, Dublin $2 ;{ }^{2} \mathrm{UCD}$ School of Agriculture, \\ Food Science and Veterinary Medicine, University College Dublin, Belfield, Dublin 4, Ireland
}

Received April 14, 2006; Accepted June 2, 2006

\begin{abstract}
The enhanced Semliki Forest virus vector (SFV10-E), an RNA-based suicide expression vector system, expresses foreign genes at levels up to $10 \mathrm{x}$ higher than the original SFV10 vector. This vector has been used previously to express interleukin-12 for a tumour treatment study in a $\mathrm{BALB} / \mathrm{c}$ murine model. Interleukin-18, an IFN- $\gamma$-inducing cytokine, plays a key role in the early induction of $\mathrm{T}$ helper1 (Th1) cell-mediated immune responses in addition to antiangiogenic activity. In this study, the murine IL-18 gene along with an Ig-kappa leader sequence was cloned into the SFV10-E vector. The pSFV10-E-IL-18 construct was characterised in vitro for levels of expression and secretion, and the production of biologically active IL-18 was confirmed. An in vivo tumour treatment study using high titre rSFV10-E-IL-18 virus-like particles to treat subcutaneous K-BALB and CT26 tumours in BALB/c mice demonstrated therapeutic efficacy including the disappearance of tumour cells in a minority of treated animals. Tumour regression was associated with induction of avascular and suppurative necrosis.
\end{abstract}

\section{Introduction}

IL-18 belongs to the IL-1 family of cytokines and shares structural homology with IL-1. Like IL-1 it lacks a conventional signal sequence but has its own receptor. It is produced intracellularly as an inactive $24 \mathrm{kDa}$ precursor and must be cleaved by caspase- 1 to form the mature bioactive molecule. A wide range of cells including activated macrophages, dendritic cells, Kupffer cells, osteoblasts and epithelial cells produces the mature $18.3 \mathrm{kDa}$-protein. IL-18 promotes $\mathrm{NK}$ and $\mathrm{T}$ cells to secrete IFN- $\gamma$ and the granulocytemacrophage colony stimulating factor (GM-CSF) $(1,2)$.

Correspondence to: Professor G.J. Atkins, Virus Group, Department of Microbiology, Moyne Institute, Trinity College, Dublin 2, Ireland

E-mail: gatkins@tcd.ie

Key words: interleukin-18, Semliki Forest virus vector, tumour inhibition, K-BALB tumours, CT26 tumours
IL-18 has been shown to be effective in the treatment of a variety of murine tumours, either alone or in combination with IL-12. Transduction of the IL-18 gene induces antitumour activity by the augmentation of NK-cell activity, the production of IFN $-\gamma$ by activated T-cells, and the inhibition of angiogenesis (3-12).

IL-18 is associated with Th1 response and, as such, can be a mediator of tissue injury and pathology (2). Local administration of the cytokine mediated by vectors, including viruses, dendritic cells and plasmids, has proved successful in mediating anti-tumour activity $(9,10)$, but the systemic administration of IL-18 has been shown to be associated with toxicity $(2,12)$.

The Semliki Forest virus (SFV) vector system induces high-level transient RNA-based suicidal expression and, like other alphaviruses, has recently been developed both as a cytolytic anti-cancer agent and for the construction of prototype anti-tumour vaccines (13-15). This system consists of an expression vector RNA with which foreign genes can be expressed, and two helper vectors that encode the structural protein genes. Co-transfection of all three RNA transcripts into mammalian cells results in the release of recombinant virus-like particles (VLPs) coding for the foreign gene (16-18). As the resulting VLP progeny contain only the recombinant RNA and lack the viral structural genes, they are capable of only one round of replication. Such recombinant particles (rSFV) are 'suicide' particles in that they undergo one round of multiplication on infection but are unable to proceed further because the structural genes are missing and the RNA cannot be encapsulated. SFV also induces apoptosis of infected cells (19), which means that the virus does not persist in tissue after infection (20).

Recently, a new SFV-based enhanced expression vector, SFV10-E, has been developed. Cells transfected with this vector have been shown to produce up to 10x more foreign protein than the original SFV expression vector. This vector was used in our previous study to express IL-12 for tumour treatment in mouse models (21). This enhanced expression vector contains the $\mathrm{N}$-terminal 34-amino acid sequence of the SFV capsid enhancer protein and vectors encoding this minimal enhancer sequence express the foreign gene as a fusion protein. In order to obtain high levels of expression of heterologous protein without the additional $\mathrm{N}$-terminal $\mathrm{C}$ residues, the $2 \mathrm{~A}$ autoprotease from foot-and-mouth disease virus (FMDV) was inserted as a linker between the enhancer 
and foreign gene sequences. The capsid translational enhancer sequence was also included upstream of the envelope proteins (together with the $2 \mathrm{~A}$ autoprotease of FMDV) in the pSFV-HelperS2 construct to ensure comparable levels of expression of the envelope and C proteins (21).

In this study, we cloned the murine IL-18 gene into SFV10 -E to produce a biologically active mature protein and measured expression of the protein in cultured BHK-21 and K-BALB cells. We examined the anti-tumour effect of pSFV10-E-IL-18 in two different tumour models in immunocompetent BALB/c mice. BHK-21 and K-BALB fibrosarcoma cells were used for virus particle titration and cell culture studies. K-BALB cells are murine sarcoma virus transformed mouse fibroblasts that overexpress the K-ras oncogene and form aggressive localised syngeneic tumours in immunocompetent BALB/c mice on subcutaneous (s.c.) injection $(22,23)$. CT26 cells are murine colon adenocarcinoma cells, which form localised tumours of low immunogenicity in BALB/c mice after s.c. injection (24). We showed that the treatment of K-BALB and CT26 tumours with rSFV10-E-IL-18 VLPs induced significant inhibition of tumour growth. Regression of tumour growth was associated with increased levels of avascular and suppurative necrosis. Tumour cells were absent at sites of tumour regression in a minority of treated animals.

\section{Materials and methods}

Cell culture. The BHK-21, K-BALB, and CT26 cell lines were obtained from the American Type Culture Collection (MD, USA). The BHK cell line, sBHK, was a gift from Professor P. Liljeström (Microbiology and Tumorbiology Center, Karolinska Institute, Stockholm, Sweden). BHK-21 and sBHK cells were cultured in BHK-21 medium supplemented with 5\% newborn calf serum, $10 \%$ tryptose phosphate broth, $20 \mathrm{mM}$ HEPES buffer, $100 \mathrm{U} / \mathrm{ml}$ penicillin, $100 \mu \mathrm{g} / \mathrm{ml}$ streptomycin, and $2 \mathrm{mM}$ L-glutamine. K-BALB and CT26 tumour cells were grown in Dulbecco's modified Eagle's medium (DMEM) supplemented with 10\% (v/v) newborn calf serum, $100 \mathrm{U} / \mathrm{ml}$ penicillin, $100 \mu \mathrm{g} / \mathrm{ml}$ streptomycin, and $10 \mathrm{mM}$ sodium pyruvate. Splenocytes were cultured in RPMI-1640 medium containing 10\% foetal bovine serum (FBS), $10 \mathrm{mM}$ HEPES, $2 \mathrm{mM}$ glutamine, $100 \mathrm{U} / \mathrm{ml}$ penicillin, $100 \mu \mathrm{g} / \mathrm{ml}$ streptomycin, $10 \mathrm{mM}$ sodium pyruvate, $1 \%$ non-essential amino acids, $50 \mu \mathrm{M} ß$-mercaptoethanol.

Mice. Specific pathogen-free 4 to 6-week old female BALB/c mice (Harlan, UK) were maintained in accordance with the principles outlined in S1 17/94 European Communities regulations 1994 , for the care and use of laboratory animals.

Construction of the pSFV10-E-IL-18 vector. To construct the pSFV10-E-IL-18 vector, IL-18 cDNA was amplified from total RNA of BALB/c mouse splenocytes using oligonucleotide primers and a reverse transcription kit (Promega, UK). The primers had terminal HindIII restriction enzyme sites (bold) and the sequences were: 5'CCC AAG CTT AAC TTT GGC CGA CTT3' (forward) and 5'CCC AAG CTT CTA ACT TTG ATG TAA G3' (reverse). Amplification was performed using the TripleMaster Taq PCR system
(Eppendorf, UK). The PCR product was cloned into the pSecTag2 B expression vector (Invitrogen, UK). The pSecTag2 B plasmid was used to add Ig-kappa signal sequence to the $\mathrm{N}$-terminus of the IL-18 gene to induce the expression and secretion of the functional IL-18 protein. The IL-18 gene along with the leader sequence from the pSecTag2 B-IL18 construct was PCR amplified using the following primers: 5'TTA TAA TAC CCG GGA TGG AGA CAG ACA CA3' (forward) and 5'CTA TAC CCG GGA AGC TTC TAA CTT TGA TGT A3' (reverse). The pSFV10-E vector and the Ig-К-IL-18 insert were digested with Xma1 and the insert was ligated into the XmaI linearised vector using T4 DNA Quick Ligase to obtain pSFV10-E- Igк-IL-18.

Production of rSFV10-E-IL-18 virus-like particles. The SFV split-helper vector system that encompasses the packaging vectors pSFV-HelperS2 (encoding the envelope proteins) and pSFV-CS219A (encoding the capsid protein), and pSFVEGFP and pSFV10-E, was obtained from Professor P. Liljeström. For the production of rSFV10-E-IL-18 VLPs, the methods described previously were used (21). In vitro transcripts of the IL-18 construct and the helper vectors (rSFV-Helper S2 and rSFV-Helper CS219A) were coelectroporated into sBHK cells. After incubating the cells for $36 \mathrm{~h}$ at $33^{\circ} \mathrm{C}$ in $5 \% \mathrm{CO}_{2}$, the medium containing the VLPs was harvested, clarified by centrifugation, aliquoted, and stored at $-70^{\circ} \mathrm{C}$. Titration was performed by infecting the monolayers of BHK-21 cells and immunofluorescence was carried out using rat anti-mouse IL-18 antibodies (Pharmingen) $18 \mathrm{~h}$ post infection. To obtain high titer stocks of rSFV10-E-IL-18 VLPs, the supernatant was concentrated by ultracentrifugation through a sucrose cushion as described previously (21).

Secretion of IL-18 by cultured cells infected with rSFV-10-E$I L-18$. Secretion levels of IL-18 from infected BHK-21 and K-BALB cells were analysed by ELISA using the OptEIA ${ }^{\mathrm{TM}}$ Set Mouse IL-18 kit (BD Biosciences Pharmingen) according to the manufacturer's instructions. The cell supernatants were collected 12, 24, 48, and $72 \mathrm{~h}$ after mock (TNE) infection, rSFV-EGFP or rSFV10-E-IL-18 VLP infection at a multiplicity of infection (MOI) of 10 .

The biological activity of the secreted IL-18 from both cell lines was assayed using mouse splenocytes ( $\mathrm{T}$ cells). The splenocytes were incubated with supernatants of mock or rSFV-IL-18 VLP-infected BHK-21 or K-BALB cells collected at $24 \mathrm{~h}$. Naïve and Concavalin A (Con A) $(1 \mu \mathrm{g} / \mathrm{ml})$ treated splenocytes were used as controls since Con A is an inducer of IFN- $\gamma$ by T-cells. The supernatant from the splenocytes was collected at $12,24,48$, and $72 \mathrm{~h}$ and assayed for IFN- $\gamma$ as before (21).

Immunofluorescence of rSFV10-E-IL-18 VLP-infected BHK-21 cells (18 h post-infection) was carried out using goat anti-rat IL-18 antibody (R\&D Systems, UK).

Treatment of $K-B A L B$ and CT26 tumours using rSFV10-E$I L-18$ VLPs. Tumours were induced subcutaneously into the right flank by injecting $10^{6}$ cells as described previously (21). Tumours were measured daily using a linear caliper and average tumour diameter was calculated as the square root of 
$\mathbf{A}$
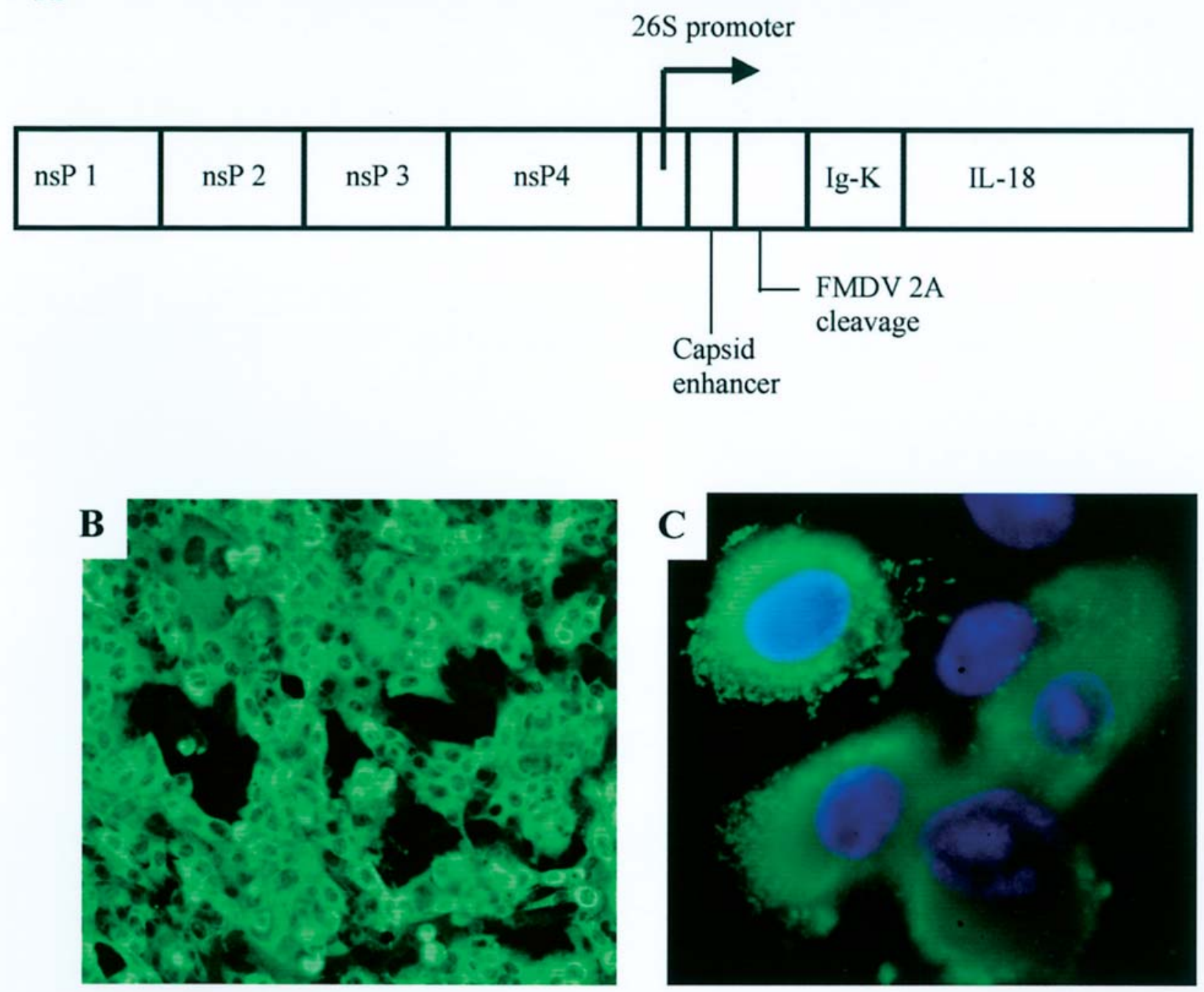

Figure 1. (A) Schematic representation of the pSFV10-E-IL-18 construct. The coding sequences of IL-18 gene with upstream Ig-kappa transport signal sequences were cloned into the MCS of pSFV10-E. The 26S sub-genomic promoter, Kozak, the capsid enhancer and FMDV 2A cleavage-site of pSFV10-E regulated the expression of IL-18. Original magnification $\mathrm{x} 40$. (B and C) Immunofluorescence staining for IL-18. BHK-21 cells were infected with rSFV10E-IL-18 and examined $18 \mathrm{~h}$ post infection. Original magnification x40 (B), x200 (C).

the product of two perpendicular measurements, assuming spherical shape. Treatment began when the size of the established tumours reached $4 \mathrm{~mm}$ diameter. The tumours were treated every alternate day with a total of six intratumoural injections of $50 \mu 1$ of TNE buffer alone, TNE buffer containing rSFV-EGFP (virus control), and rSFV10E-IL-18 VLPs at a titre of $4 \times 10^{9}$ IU for each injection. Efficient therapeutic effects were obtained using this regimen in our previous tumour study on IL-12 (21). Two sets of animals for each treatment group were used for each tumour cell line. The first group was assayed for survival and the second group assayed for histopathology. Mice were euthanised one day following the final treatment or when the tumour size reached $10 \%$ of body weight $(15 \mathrm{~mm})$.

Histopathology. For histopathology, $3 \mu \mathrm{m}$ sections of formaldehyde-fixed, paraffin-embedded tumours were stained routinely with $H \& E$. Separate sections containing three different areas in each tumour were examined in a blinded manner and the following parameters were assessed: Tumour cell morphology, heterogeneity, mitotic index, infiltration, ulceration, necrosis, haemorrhage, thrombosis, and leucocytic infiltrates.

Statistical analysis. The statistical data analysis of tumour treatment groups was performed by one-way repeated measures ANOVA. The probability (P-value) of each group was calculated using Tukey's multiple comparison post-test. All statistical analyses were made using the GraphPad Prism4 program and a $\mathrm{P}$-value $<0.05$ was considered significant.

\section{Results}

IL-18 expression in BHK-21 and $K-B A L B$ cells. The murine IL-18 gene was cloned into the enhanced expression vector pSFV10-E to obtain the pSFV10-E-IL-18 vector that contains the Igא leader sequence (Fig. 1A). Immuno- 
A

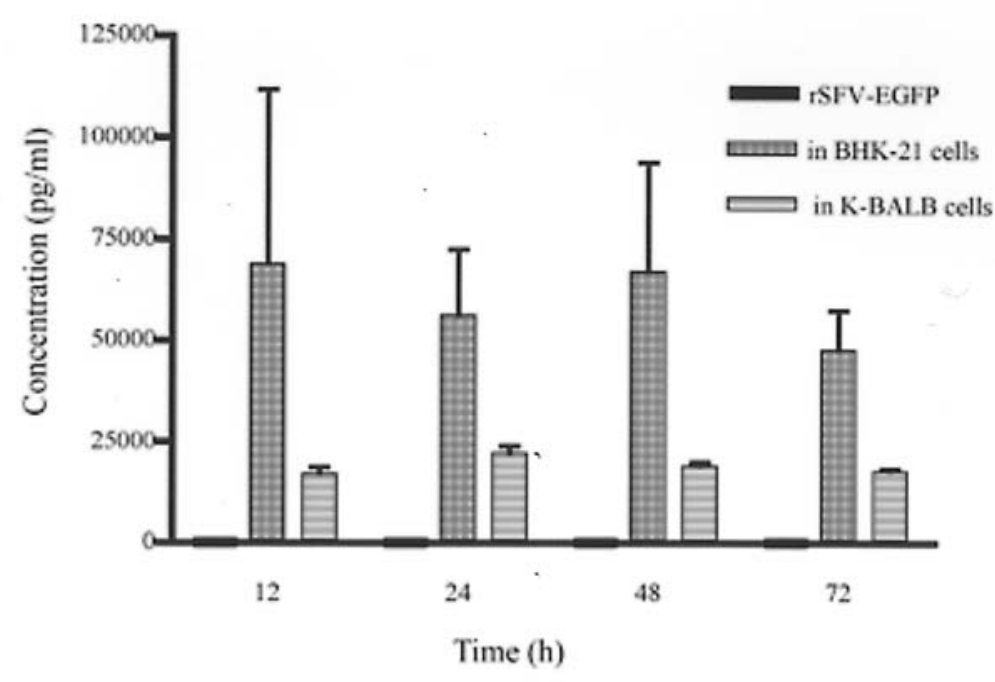

B

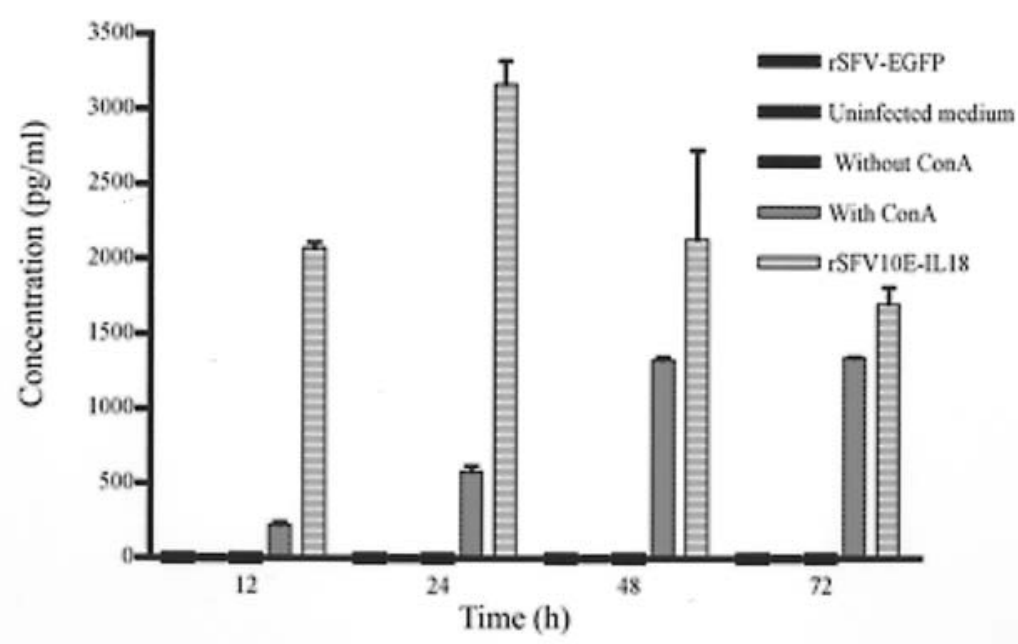

Figure 2. (A) IL-18 expression levels in the supernatants of rSFV10-E-IL-18 infected BHK-21 and K-BALB cells. The values shown represent IL-18 secretion from $2.5 \times 10^{5}$ cells as measured by ELISA. (B) Biological activity of murine IL-18 secreted by K-BALB cells. Using ELISA the production of IFN- $\gamma$ from murine splenocytes $\left(10^{6}\right.$ cells) treated with supernatants from rSFV10-E-IL-18 infected K-BALB cells was measured. Supernatants from control groups (rSFV-EGFP, uninfected, and without Con A) were unable to induce IFN- $\gamma$ production.

fluorescence staining of rSFV10-E-IL-18-infected BHK-21 cells using IL-18 specific antibody qualitatively confirmed IL-18 expression (Fig. 1B). Estimation of IL-18 in the supernatants of BHK-21 and K-BALB cells infected with rSFV10-E-IL-18 was carried out at 12, 24, 48 and $72 \mathrm{~h}$ after infection using ELISA (Fig. 2A). The levels of IL-18 expression were 2-3x higher in BHK-21 cells than in KBALB cells. Overall expression levels were similar to those recorded in our previous study using IL-12 (21). IL-18 expression was absent in the supernatants from mockinfected control BHK-21 and K-BALB cells.

Biological functionality of secreted IL-18. Using ELISA the production of IFN- $\gamma$ from the $24 \mathrm{~h}$ supernatants of murine splenocytes treated with supernatants from rSFV10-E-IL-18infected K-BALB cells was measured. The results showed that the supernatants of K-BALB cells infected with rSFV10E-IL-18 stimulated splenocytes to produce higher levels of IFN- $\gamma$ than Concavalin A treatment, indicating the production of functionally active IL-18 (Fig. 2B).

Treatment of K-BALB and CT26 tumours using rSFV10-E$I L-18$. Tumour growth was monitored daily and mice were euthanised one day following final treatment or when the average tumour diameter reached $15 \mathrm{~mm}$. The average time taken to reach this size for a group of mice represented the survival time and mice showing complete tumour regression were considered survivors.

The growth of K-BALB and CT26 tumours was inhibited following treatment with rSFV10-E-IL-18 and the levels of growth inhibition were significant compared to the controls. A complete regression of tumours and survival occurred in 2 

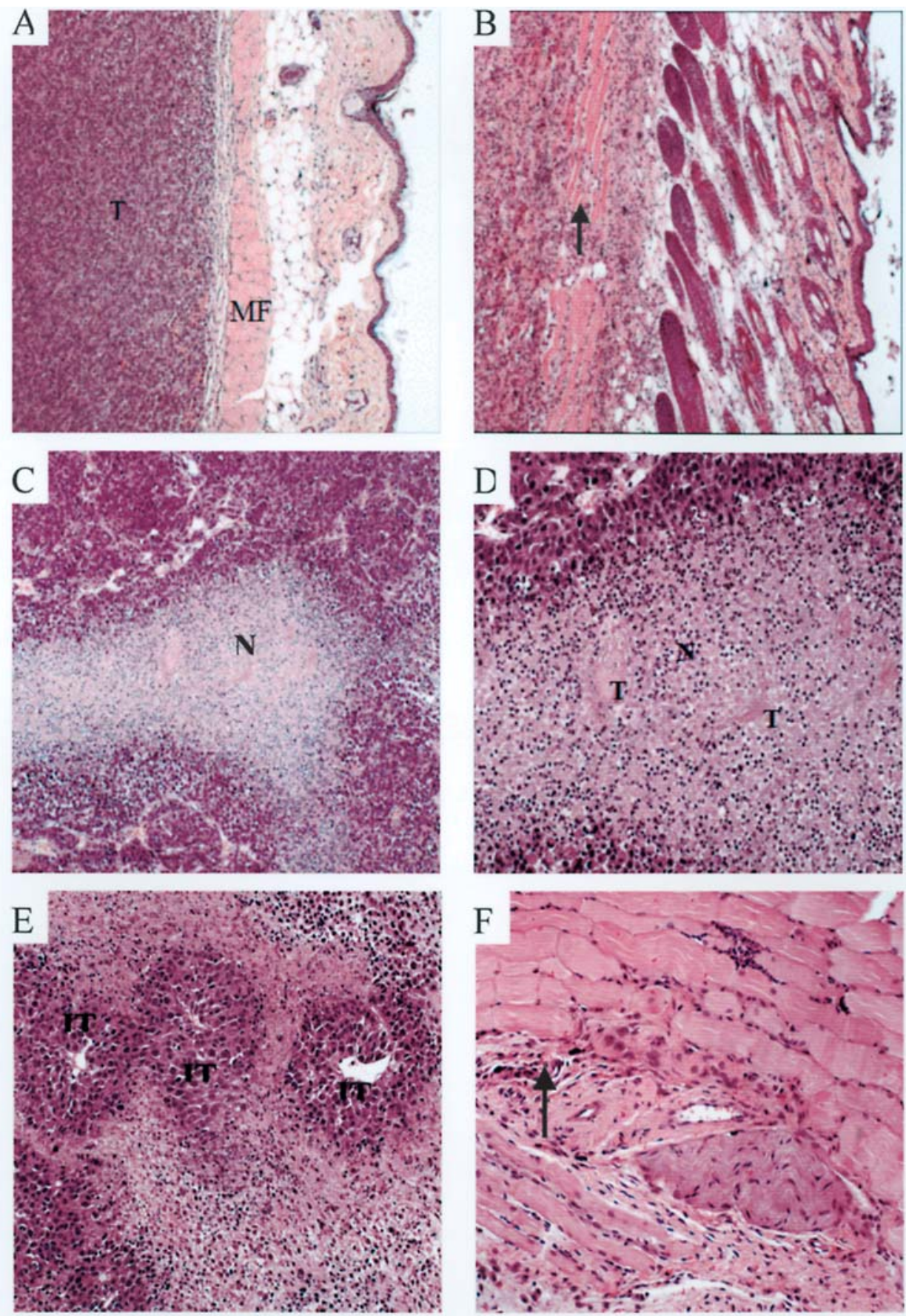

Figure 3. (A and B) K-BALB tumours, one day following final treatment with TNE. (A) Dense aggregate of tumour cells (T) in the subcutis. The epidermis, dermis and panniculus muscle fibres (MF) are intact. H\&E, x100. (B) Invasion of tumour cells through the panniculus muscle (arrow) into the deep dermis. $\mathrm{H} \& \mathrm{E}, \mathrm{x} 100$. (C and D) CT26 tumours, one day following final treatment with rSFV10-E-IL-18. (C) Centrally located area of suppurative necrosis (N). H\&E, x100. (D) Higher magnification of (C) showing fibrin thrombi (T) and neutrophils. H\&E, x200. (E and F) K-BALB tumours, one day following final treatment with rSFV10-E-IL-18. (E) Islands of intact tumour cells (IT) around thin-walled blood vessels and avascular necrosis in marginal areas. H\&E, x100. (F) Fibrous scarring, low-grade infiltrates of lymphocytes and macrophages (arrow) and regenerating skeletal muscle myofibers. No tumour cells are visible. $\mathrm{H} \& \mathrm{E}, \mathrm{x} 200$.

of 6 mice with K-BALB tumours and in 2 of 6 mice with CT26 tumours. The control (TNE or rSFV-EGFP) treated groups showed a similar tumour growth with no survivors (Table I).

Histopathology. The control tumours were located in the subcutis and were composed of densely cellular, expansive masses with no evidence of encapsulation. Focal necrosis, ulceration and invasion of panniculus muscle fibres were common (Fig. 3A and B). Areas of avascular and suppurative necrosis with fibrin thrombi and haemorrhage were more prominent in rSFV10-E-IL-18 treated tumours than in the control tumours. Neutrophils and macrophages were located in areas of necrosis and lymphocytes were sparsely distributed in peripheral areas at the interface with surrounding tissues (Fig. 3C-E). Tumour cells were not detected at the sites of induction of CT26 tumours in 2 mice following local treatment with rSFV10-E-IL-18 where small 
Table I. Inhibition of tumour growth by rSFV10-E-IL-18 treatment.

\begin{tabular}{|c|c|c|c|c|}
\hline $\begin{array}{l}\text { Tumour } \\
\text { model }\end{array}$ & Treatment group ${ }^{\mathrm{a}}$ & $\begin{array}{l}\text { Median survival (days) } \\
\text { (No. of survivors) }^{\mathrm{c}}\end{array}$ & $\begin{array}{l}\text { Average tumour } \\
\text { diameter }(\mathrm{mm}) \\
\text { (std. error) }\end{array}$ & Probability $^{\mathrm{f}}$ \\
\hline \multirow[t]{3}{*}{ K-BALB } & $\mathrm{TNE}^{\mathrm{e}}$ & $22(0)$ & $11.9( \pm 0.66)$ & \\
\hline & rSFV-EGFP (4x109 IU/dose) & $23(0)$ & $11.4( \pm 0.65)$ & $>0.05$ \\
\hline & rSFV10-E-IL18 (4x1099 IU/dose) & $32(2)$ & $6.3( \pm 0.42)$ & $<0.01$ \\
\hline \multirow[t]{3}{*}{ CT26 } & TNE & $24(0)$ & $11.0( \pm 0.67)$ & \\
\hline & rSFV10-EGFP (4x109 IU/dose) & $28(0)$ & $9.8( \pm 0.69)$ & $>0.05$ \\
\hline & rSFV10-E-IL18 (4x1099 IU/dose) & $35(2)$ & $5.9( \pm 0.45)$ & $<0.01$ \\
\hline
\end{tabular}

${ }^{a} \mathrm{Six} \mathrm{BALB/c} \mathrm{mice} \mathrm{per} \mathrm{treatment} \mathrm{group} \mathrm{were} \mathrm{used} \mathrm{for} \mathrm{all} \mathrm{experiments;}{ }^{\mathrm{b}}$ mice were sacrificed when the tumour reached $15 \mathrm{~mm}$ diameter and the time taken to reach this size is the survival time; ${ }^{c}$ mice which had complete and permanent tumour regression; ${ }^{\mathrm{d}}$ average tumour diameter for each group was calculated at their appropriate median survival time; ${ }^{\mathrm{e}}$ average of the control groups treated with TNE buffer only; ${ }^{\mathrm{f}}$ the probability value is based on the average tumour diameter at their median survival time compared with that of the TNE treated (control) groups.

cutaneous nodules were characterised by fibrosis, chronic inflammation and atrophic and regenerative changes in skeletal muscle myofibers (Fig. 3F).

\section{Discussion}

Several cytokine genes introduced intratumourally in mice have shown anti-tumour effects. The strong anti-tumour properties of IL-12 and IL-18 have been previously reported using murine renal cell carcinoma and bladder cancer models $(4,5)$. We have previously shown the anti-tumour effect of IL-12-expressing SFV VLPs in K-BALB and CT26 murine models (21); in this study extended this to IL-18.

IL-18 is first synthesised as a leaderless precursor, and it requires a signal sequence for extracellular secretion of the mature protein. We constructed a pSFV10-E-IL-18 vector containing the Igא leader sequence to regulate the expression of mature IL-18. It has been reported that direct injection of an IL-18 adenoviral vector into mice with MCA205 sarcoma elicits an effective immune response. The dose of IL-18 administered correlated with the resulting level of serum IFN $-\gamma$ and the level of anti-tumour activity (25). The rSFV10-E-IL-18 vector constructed in the present study showed higher levels of expression in the supernatants of infected BHK-21 cells than in the supernatants of infected KBALB cells. These differences probably reflected different levels of infectivity for the two cell lines and varying abilities to secrete the expressed protein. To determine the bioactivity of secreted IL-18, we induced mouse splenocytes (T-cells) for IFN- $\gamma$ production and the results confirmed that the IL-18 produced by rSFV10-E-IL-18 in K-BALB cells is biologically functional. The IL-18 construct with enhanced expression ability, which contains the Igא leader sequence, induced secretion of large amounts of IFN- $\gamma$. These observations are consistent with a previous study where successful immunogene tumour therapy was achieved using a plasmid expression vector along with pro-IL-18 sequences that also contained the Igк leader sequence (26).
In this study we showed that the treatment of K-BALB and CT26 tumours with rSFV10-E-IL-18 induced a significant inhibition of tumour growth. Complete tumour regression occurred in a minority of treated animals. The findings resemble those obtained in a previous study of $\mathrm{K}$ BALB and CT26 tumours treated with rSFV10-E-IL-12 (21) and showed that IL-18 and IL-12 have potent anti-tumour effects when expressed from the enhanced expression SFV vector.

\section{Acknowledgements}

We thank Alex Whelan for assistance with histopathological studies, Brian Cloak for assistance with photomicrography and Dorothy Mooney for technical support. We also thank Professor Peter Liljeström of the Microbiology and Tumorbiology Center, Karolinska Institute, Stockholm for providing the SFV expression vectors. This study was supported by Cancer Research Ireland, the European Union and Science Foundation Ireland.

\section{References}

1. Okamura H, Tsutsui H, Kashiwamura S, Yoshimoto T and Nakanishi K: IL-18. In: Cytokine Reference. Vol. 1. Academic Press, San Francisco, 2001.

2. Dinarello CA and Fantuzzi G: Interleukin-18 and host defense against infection. J Infect Dis 187: 370-384, 2003.

3. Micallef MJ, Yoshida K, Kawai S, et al: In vivo antitumor effects of murine interferon-gamma-inducing factor/interleukin18 in mice bearing syngeneic Meth A sarcoma malignant ascites. Cancer Immunol Immunother 43: 361-367, 1997.

4. Yamanaka K, Hara I, Nagai H, et al: Synergistic antitumor effects of interleukin-12 gene transfer and systemic administration of interleukin-18 in a mouse bladder cancer model. Cancer Immunol Immunother 48: 297-302, 1999.

5. Hara I, Nagai $\mathrm{H}$, Miyake $\mathrm{H}$, et al: Effectiveness of cancer vaccine therapy using cells transduced with the interleukin-12 gene combined with systemic interleukin-18 administration. Cancer Gene Ther 7: 83-90, 2000

6. Oshikawa K, Shi F, Rakhmilevich AL, Sondel PM, Mahvi DM and Yang NS: Synergistic inhibition of tumor growth in a murine mammary adenocarcinoma model by combinational gene therapy using IL-12, pro-IL-18, and IL-1beta converting enzyme cDNA. Proc Natl Acad Sci USA 96: 13351-13356, 1999. 
7. Tatsumi T, Huang J, Gooding WE, et al: Intratumoral delivery of dendritic cells engineered to secrete both interleukin (IL)-12 and IL-18 effectively treats local and distant disease in association with broadly reactive Tc1-type immunity. Cancer Res 63: 6378-6386, 2003.

8. Coughlin CM, Salhany KE, Wysocka M, et al: Interleukin-12 and interleukin-18 synergistically induce murine tumor regression which involves inhibition of angiogenesis. J Clin Invest 101: 1441-1452, 1998.

9. Han M, Zheng S, Yu J, Peng J, Guo Q and Wang J: Study on interleukin-18 gene transfer into human breast cancer cells to prevent tumorigenicity. J Zhejiang Univ Sci 5: 472-476, 2004.

10. Yamanaka R, Tsuchiya N, Yajima $\mathrm{N}$, et al: Induction of an antitumor immunological response by an intratumoral injection of dendritic cells pulsed with genetically engineered Semliki Forest virus to produce interleukin-18 combined with the systemic administration of interleukin-12. J Neurosurg 99: 746-753, 2003.

11. Hara S, Nagai H, Miyake H, et al: Secreted type of modified interleukin-18 gene transduced into mouse renal cell carcinoma cells induces systemic tumor immunity. J Urol 165: 2039-2043, 2001.

12. Nakamura S, Otani, T, Ijiri Y, Motoda R, Kurimoto M and Orita K: IFN- $\gamma$-dependent and independent mechanisms in adverse effects caused by concomitant administration of IL-18 and IL-12. J Immunol164: 3330-3336, 2000.

13. Lundstrom K: Alphavirus vectors for gene therapy applications. Curr Gene Ther 1: 19-29, 2001.

14. Atkins GJ, Smyth JW, Fleeton MN, Galbraith SE and Sheahan BJ: Alphaviruses and their derived vectors as anti-tumor agents. Curr Cancer Drug Targets 4: 597-607, 2004.

15. Karlsson GB and Liljestrom P: Delivery and expression of heterologous genes in mammalian cells using self-replicating alphavirus vectors. Methods Mol Biol 246: 543-557, 2004.

16. Berglund P, Sjoberg M, Garoff H, Atkins GJ, Sheahan BJ and Liljestrom P: Semliki Forest virus expression system: production of conditionally infectious recombinant particles. Biotechnology 11: 916-920, 1993.

17. Smerdou C and Liljestrom P: Two-helper RNA system for production of recombinant Semliki forest virus particles. J Virol 73: 1092-1098, 1999.
18. Lundstrom K, Schweitzer C, Rotmann D, Hermann D, Schneider EM and Ehrengruber MU: Semliki Forest virus vectors: efficient vehicles for in vitro and in vivo gene delivery. FEBS Lett 504: 99-103, 2001.

19. Glasgow GM, McGee MM, Tarbatt CJ, Mooney DA, Sheahan BJ and Atkins GJ: The Semliki Forest virus vector induces p53independent apoptosis. J Gen Virol 79: 2405-2410, 1998.

20. Morris-Downes MM, Phenix KV, Smyth J, et al: Semliki Forest virus-based vaccines: persistence, distribution and pathological analysis in two animal systems. Vaccine 19: 19781988, 2001.

21. Chikkanna-Gowda CP, Sheahan, BJ, Fleeton, MN and Atkins GJ: Regression of mouse tumours and inhibition of metastases following administration of a Semliki Forest virus vector with enhanced expression of IL-12. Gene Ther 12: 1253-1263, 2005.

22. Aaronson SA and Weaver CA: Characterization of murine sarcoma virus (Kirsten) transformation of mouse and human cells. J Gen Virol 13: 245-252, 1971.

23. Stephenson JR and Aaronson SA: Antigenic properties of murine sarcoma virus-transformed BALB-3T3 nonproducer cells. J Exp Med 135: 503-515, 1972.

24. Brattain, MG, Strobel-Stevens J, Fine D, Webb M and Sarrif AM: Establishment of mouse colonic carcinoma cell lines with different metastatic properties. Cancer Res 40: 2142-2146, 1980.

25. Osaki T, Hashimoto W, Gambotto A, et al: Potent antitumor effects mediated by local expression of the mature form of the interferon-gamma inducing factor, interleukin-18 (IL-18). Gene Ther 6: 808-815, 1999.

26. Yoshimura K, Hazama S, Iizuka N, Yoshino S, Yamamoto K, Muraguchi M, Ohmoto Y, Noma T and Oka M: Successful immunogene therapy using colon cancer cells (colon 26) transfected with plasmid vector containing mature interleukin$18 \mathrm{cDNA}$ and the Igkappa leader sequence. Cancer Gene Ther 8: 9-16, 2001. 